\title{
On Implicit Algorithms for Solving Variational Inequalities
}

\author{
Eman Al-Shemas \\ Department of Mathematics, College of Basic Education, Main Campus, Shamiya, Kuwait \\ Email: eh.alshemas@paaet.edu.kw,emanalshemas@gmail.com
}

Received October 8, 2012; revised November 15, 2012; accepted November 23, 2012

\begin{abstract}
This paper presents new implicit algorithms for solving the variational inequality and shows that the proposed methods converge under certain conditions. Some special cases are also discussed.
\end{abstract}

Keywords: Variational Inequalities; Fixed Point Methods; Predictor-Corrector Methods

\section{Introduction}

Variational inequality theory, introduced by Stampaccia [1], provides simple and unified framework to study a large number of problems arising in finance, economics, transportation, network and structural analysis, elasticity and optimization. Variational inequality theory, was emerged as an interesting and fascinating branch of applicable mathematics with a wide range of applications in unrelated linear and nonlinear problems.

The projection method provides important tools for finding the approximate solution of variational inequalities. This method is due to Lions and Stampacchia [2]. The main idea in this technique is to establish the equivalence between the variational inequalities and the fixed-point problem by using the concept of projection. This alternative formulation has played a significant part in developing various projection-type methods, the implicit iterative method, and the extra-gradient method which is due to Korpelevich [3], for solving the variational inequalities.

In this paper, we use the equivalent fixed point formulation to suggest and analyze some new implicit iterative methods for solving the variational inequalities. We have shown that these new implicit methods include the unified implicit, the proximal point and the modified extra gradient methods of Noor et al. [4,5], Noor [6] and the extra gradient method of Korpelevich [3] as special cases. We consider the convergence analysis of these methods under certain conditions.

\section{Preliminaries}

Let $H$ be a real Hilbert space whose inner product and norm are denoted by $\langle. .$.$\rangle and \|$.$\| respectively. Let K$ be a nonempty closed convex subset in $H$.

For a given nonlinear operator $T: H \rightarrow H$, we consider the problem of finding $u \in K$ such that

$$
\langle T u, v-u\rangle \geq 0, \forall v \in K,
$$

Problem (1) is called the variational inequality, introduced and studied by Stampacchia [1]. For more information about applications,numerical methods and other aspects of variational inequalities, one may refer to [112].

First we recall the following well-known results and concepts.

Lemma 1. Let $K$ be a nonempty, closed, and convex set in $H$. Then, for a given $z$ in $H, u \in K$ satisfies the inequality

$$
\langle u-z, v-u\rangle \geq 0, \forall v \in K,
$$

if and only if

$$
u=P_{K} z,
$$

where $P_{K}$ is the projection of $H$ onto the closed and convex set $K$.

It is well known that the projection operator $P_{K}$ is nonexpansive, that is

$$
\left\|P_{K} u-P_{K} v\right\| \leq\|u-v\|, \forall u, v \in H .
$$

Now if $K$ is a nonempty, closed and convex subset in $H$, then Problem (1) is equivalent to the existence of $u \in K$ such that

$$
0 \in T u+N_{K}^{P}(u),
$$

where $N_{K}^{P}(u)$ denotes the normal cone of $K$ at $u$. Problem (2) is called the variational inclusion problem associated with the variational inequality (1).

Definition 1. An operator $T: H \rightarrow H$ is said to be strongly monotone if and only if there exists a constant $\alpha>0$ such that

$$
\langle T u-T v, u-v\rangle \geq \alpha\|u-v\|^{2}, \forall u, v \in H,
$$

and Lipschitz continuous if there exists a constant $\beta>0$ 
such that

$$
\|T u-T v\| \leq \beta\|u-v\|, \forall u, v \in H .
$$

\section{Main Results}

In this section, using Lemma 1, one can easily show that the variational inequality (1) is equivalent to the existence of $u \in K$ such that

$$
u=P_{K}[u-\rho T u],
$$

where $\rho>0$ is constant.

Equation (3) is a fixed point problem and will be used in suggesting some new implicit methods for solving the variational inequality (1), and this is the main motivation of this paper.

Now, using the equivalent fixed point formulation (3), one can suggest the following iterative method for solving the variational inequality (1).

Algorithm 1. For a given $u_{0} \in K$, find the approximate solution $u_{n+1}$ by the iterative scheme

$$
u_{n+1}=P_{K}\left[u_{n}-\rho T u_{n}\right], n=0,1,2 .
$$

Algorithm 1 is known as the projection iterative method.

For a given $\lambda \in[0,1]$, we can rewrite (3) as

$$
u=P_{K}[(1-\lambda)(u-\rho T u)+\lambda(u-\rho T u)],
$$

This fixed point formulation is used to suggest the following iterative method for solving variational inequality (1).

Algorithm 2. For a given $u_{0} \in K$, find the approximate solution $u_{n+1}$ by the iterative scheme

$$
\begin{aligned}
& u_{n+1}=P_{K}\left[(1-\lambda)\left(u_{n+1}-\rho T u_{n+1}\right)+\lambda\left(u_{n}-\rho T u_{n}\right)\right], \\
& n=0,1,2 .
\end{aligned}
$$

Note that Algorithm 2 is an implicit type iterative method and includes the implicit method of Noor [6] and the classical projection method as special cases.

In order to implement this method, we use the predictor-corrector technique. We use Algorithm 1 as the predictor and Algorithm 2 as the corrector. Consequently, we obtain the following two-step iterative method for solving the variational inequality (1).

Algorithm 3. For a given $u_{0} \in K$, find the approximate solution $u_{n+1}$ by the iterative schemes

$$
\begin{gathered}
y_{n}=P_{K}\left[u_{n}-\rho T u_{n}\right], \\
u_{n+1}=P_{K}\left[(1-\lambda)\left(y_{n}-\rho T y_{n}\right)+\lambda\left(u_{n}-\rho T u_{n}\right)\right], \\
n=0,1,2 .
\end{gathered}
$$

Algorithm 3 is a new two-step implicit iterative method for solving the variational inequality (1). For $\lambda=0$, Algorithm 3 reduces to the following iterative method for solving variational inequality (1).

Algorithm 4. For a given $u_{0} \in K$, find the approximate solution $u_{n+1}$ by the iterative schemes

$$
\begin{gathered}
y_{n}=P_{K}\left[u_{n}-\rho T u_{n}\right], \\
u_{n+1}=P_{K}\left[y_{n}-\rho T y_{n}\right], n=0,1,2,
\end{gathered}
$$

which is known as the modified double projection method, Noor [6].

For $\lambda=1$, Algorithm 3 reduces to algorithm 1 for solving variational inequality (1).

This shows that Algorithm 3 is a unified implicit method and includes the previously known implicit and predictor-corrector methods as special cases.

Now for a given $\mu \in[0,1]$ and $\lambda \in[0,1]$, we can rewrite (3) as

$$
u=P_{K}[\mu(u-u)+u-\rho T u+\lambda \rho(T u-T u)],
$$

For $\mu=\lambda$, the fixed point formulation (7) reduces to the fixed point formulation (4).

Now we use (7) to suggest the following iterative methods for solving variational inequality (1).

Algorithm 5. For a given $u_{0} \in K$, find the approximate solution $u_{n+1}$ by the iterative scheme

$$
\begin{aligned}
& u_{n+1} \\
& =P_{K}\left[\mu\left(u_{n+1}-u_{n}\right)+u_{n}-\rho T u_{n+1}+\lambda \rho\left(T u_{n+1}-T u_{n}\right)\right], \\
& n=0,1,2 .
\end{aligned}
$$

Note that Algorithm 5 is an implicit type iterative method and includes the implicit method of Noor et al. [7], and the classical implicit method of Korpelevich [3] as special cases.

In order to implement this method, we use the predictor-corrector technique. We use Algorithm 1 as the predictor and Algorithm 5 as the corrector. Consequently, we obtain the following iterative method for solving the variational inequality (1).

Algorithm 6. For a given $u_{0} \in K$, find the approximate solution $u_{n+1}$ by the iterative schemes

$$
\begin{gathered}
y_{n}=P_{K}\left[u_{n}-\rho T u_{n}\right], \\
u_{n+1}=P_{K}\left[\mu\left(y_{n}-u_{n}\right)+u_{n}-\rho T y_{n}+\lambda \rho\left(T y_{n}-T u_{n}\right)\right], \\
n=0,1,2 .
\end{gathered}
$$

Algorithm 6 is a new two-step implicit iterative method for solving the variational inequality (1). For $\mu=0$, Algorithm 6 reduces to the following iterative method for solving variational inequality (1).

Algorithm 7. For a given $u_{0} \in K$, find the approximate solution $u_{n+1}$ by the iterative schemes

$$
\begin{gathered}
y_{n}=P_{K}\left[u_{n}-\rho T u_{n}\right], \\
u_{n+1}=P_{K}\left[u_{n}-\rho T y_{n}+\lambda \rho\left(T y_{n}-T u_{n}\right)\right], n=0,1,2 .
\end{gathered}
$$


Algorithm 7 was studied by Noor et al. [4]. Note that for $\lambda=1$, Algorithm 7 reduces to Algorithm 1, and for $\lambda=0$, Algorithm 7 reduces to Korpelevich [3].

For $\mu=1$, Algorithm 6 reduces to the following iterative method for solving variational inequality (1), and appears to be new.

Algorithm 8. For a given $u_{0} \in K$, find the approximate solution $u_{n+1}$ by the iterative schemes

$$
\begin{gathered}
y_{n}=P_{K}\left[u_{n}-\rho T u_{n}\right], \\
u_{n+1}=P_{K}\left[y_{n}-\rho T y_{n}+\lambda \rho\left(T y_{n}-T u_{n}\right)\right], n=0,1,2 .
\end{gathered}
$$

For $\lambda=0$, Algorithm 6 reduces to the following iterative method for solving variational inequality (1), and appears to be new.

Algorithm 9. For a given $u_{0} \in K$, find the approximate solution $u_{n+1}$ by the iterative schemes

$$
\begin{gathered}
y_{n}=P_{K}\left[u_{n}-\rho T u_{n}\right], \\
u_{n+1}=P_{K}\left[\mu\left(y_{n}-u_{n}\right)+u_{n}-\rho T y_{n}\right], n=0,1,2 .
\end{gathered}
$$

For $\mu=1$, Algorithm 9 reduces to Noor [6] and for $\mu=0$, Algorithm 9 reduces to Korpelevich [3].

Now one can obtains the following iterative method for solving variational inequality (1), by using the fixed point formulation (7).

Algorithm 10. For a given $u_{0} \in K$, find the approximate solution $u_{n+1}$ by the iterative scheme

$$
\begin{aligned}
& u_{n+1} \\
& =P_{K}\left[\mu\left(u_{n}-u_{n+1}\right)+u_{n+1}-\rho T u_{n+1}+\lambda \rho\left(T u_{n+1}-T u_{n}\right)\right], \\
& n=0,1,2 .
\end{aligned}
$$

In order to implement this method, we use the predictor-corrector technique. We use Algorithm 1 as the predictor and Algorithm 10 as the corrector. Consequently, we obtain the following two-step iterative method for solving the variational inequality (1).

Algorithm 11. For a given $u_{0} \in K$, find the approximate solution $u_{n+1}$ by the iterative scheme

$$
\begin{aligned}
& \qquad y_{n}=P_{K}\left[u_{n}-\rho T u_{n}\right], \\
& u_{n+1} \\
& =P_{K}\left[\mu\left(u_{n}-y_{n}\right)+y_{n}-\rho T y_{n}+\lambda \rho\left(T y_{n}-T u_{n}\right)\right], \\
& n=0,1,2 .
\end{aligned}
$$

Algorithm 11 is a new two-step implicit iterative method for solving the variational inequality (1). For $\mu=1$, Algorithm 11 reduces to Algorithm 7 [4], and for $\mu=0$, Algorithm 11 reduces to Algorithm 8 which is a new one, as we mentioned above.

\section{Convergence}

We now consider the convergence analysis of Algorithm
3, 6 and 11, and this is the motivation of next results.

Theorem 1. Let the operator $T$ be strongly monotone with constant $\alpha>0$ and Lipschitz continuous with constant $\beta>0$. If there exists a constant $\rho>0$ such that

$$
\begin{aligned}
& 1-(1+\lambda)\left(1-2 \alpha \rho+\beta^{2} \rho^{2}\right) \\
& -\lambda \sqrt{1-2 \alpha \rho+\beta^{2} \rho^{2}}>0
\end{aligned}
$$

then, the approximate solution $u_{n+1}$ obtained from Algorithm 3 converges strongly to the exact solution $u \in K$, satisfying the variational inequality (1).

Proof. Let $u \in K$ be a solution of (1) and $u_{n+1}$ be the approximate solution obtained from Algorithm 3. Then, from (3) and (5), we have

$$
\begin{aligned}
\left\|y_{n}-u\right\| & \leq\left\|P_{K}\left[u_{n}-\rho T u_{n}\right]-P_{K}[u-\rho T u]\right\| \\
& \leq\left\|u_{n}-u-\rho\left(T u_{n}-T u\right)\right\|
\end{aligned}
$$

From the strongly monotonicity and Lipschitz continuity of the operator $T$, one obtains

$$
\begin{aligned}
& \left\|u_{n}-u-\rho\left(T u_{n}-T u\right)\right\|^{2} \\
& \leq\left\|u_{n}-u\right\|^{2}-2\left\langle u_{n}-u, T u_{n}-T u\right\rangle+\rho^{2}\left\|T u_{n}-T u\right\|^{2} \\
& \leq\left(1-2 \alpha \rho+\beta^{2} \rho^{2}\right)\left\|u_{n}-u\right\|^{2} .
\end{aligned}
$$

From (11) and (12), one obtains

$$
\begin{aligned}
\left\|y_{n}-u\right\| & \leq \sqrt{1-2 \alpha \rho+\beta^{2} \rho^{2}}\left\|u_{n}-u\right\| \\
& =\theta\left\|u_{n}-u\right\|,
\end{aligned}
$$

where

$$
\theta=\sqrt{1-2 \alpha \rho+\beta^{2} \rho^{2}}
$$

Now from (3), (6) and (13), we have

$$
\begin{aligned}
& \left\|u_{n+1}-u\right\| \\
& =\left\|P_{K}\left[(1-\lambda)\left(y_{n}-\rho T y_{n}\right)+\lambda\left(u_{n}-\rho T u_{n}\right)\right]-P_{K}[u-\rho T u]\right\| \\
& \leq(1+\lambda)\left\|y_{n}-u-\rho\left(T y_{n}-T u\right)\right\|+\lambda\left\|u_{n}-u-\rho\left(T u_{n}-T u\right)\right\| \\
& \leq(1+\lambda) \theta\left\|y_{n}-u\right\|+\lambda\left\|u_{n}-u\right\| \leq\left[(1+\lambda) \theta^{2}+\lambda \theta\right]\left\|u_{n}-u\right\| \\
& =\varphi\left\|u_{n}-u\right\|,
\end{aligned}
$$

where

$$
\begin{aligned}
\varphi & =(1+\lambda) \theta^{2}+\lambda \theta=(1+\lambda)\left(1-2 \alpha \rho+\beta^{2} \rho^{2}\right) \\
& +\lambda \sqrt{1-2 \alpha \rho+\beta^{2} \rho^{2}}
\end{aligned}
$$

From (10), it follows that $\varphi<1$. Hence, the fixed point Problem (3) has a unique solution and consequently the iterative solution $u_{n+1}$ obtained from Algorithm 3 
converges to the exact solution $u$ and satisfying the variational inequality (1). $\square$

Theorem 2. Let the operator $T$ be strongly monotone with constant $\alpha>0$ and Lipschitz continuous with constant $\beta>0$. If there exists a constant $\rho>0$ such that

$$
\begin{aligned}
& 1-\mu-\sqrt{1-2 \lambda \alpha \rho+\beta^{2} \lambda^{2} \rho^{2}} \\
& -(\mu+\rho(\lambda-1) \beta) \sqrt{1-2 \alpha \rho+\beta^{2} \rho^{2}}>0
\end{aligned}
$$

then, the approximate solution $u_{n+1}$ obtained from Algorithm 6 converges strongly to the exact solution

$u \in K$ satisfying the variational inequality (1).

Proof. Let $u \in K$ be a solution of (1) and $u_{n+1}$ be the approximate solution obtained from Algorithm 6 . Then, from (3), (8) and (13), we have

$$
\begin{aligned}
& \left\|u_{n+1}-u\right\| \\
& \leq \mu\left\|y_{n}-u_{n}\right\|+\left\|u_{n}-u-\lambda \rho\left(T u_{n}-T u\right)\right\| \\
& +\rho(\lambda-1)\left\|T y_{n}-T u\right\| \\
& \leq \mu\left\|y_{n}-u\right\|+\mu\left\|u_{n}-u\right\|+\sqrt{1-2 \lambda \alpha \rho+\beta^{2} \lambda^{2} \rho^{2}}\left\|u_{n}-u\right\| \\
& +\rho(\lambda-1) \beta \theta\left\|u_{n}-u\right\| \\
& \leq\left[\mu+\sqrt{1-2 \lambda \alpha \rho+\beta^{2} \lambda^{2} \rho^{2}}+(\mu+\rho(\lambda-1) \beta) \theta\right] \\
& \cdot\left\|u_{n}-u\right\| \\
& =\varphi\left\|u_{n}-u\right\|
\end{aligned}
$$

where

$$
\begin{aligned}
\varphi & =\mu+\sqrt{1-2 \lambda \alpha \rho+\beta^{2} \lambda^{2} \rho^{2}} \\
& +(\mu+\rho(\lambda-1) \beta) \theta \\
& =\mu+\sqrt{1-2 \lambda \alpha \rho+\beta^{2} \lambda^{2} \rho^{2}} \\
& +(\mu+\rho(\lambda-1) \beta) \sqrt{1-2 \alpha \rho+\beta^{2} \rho^{2}}
\end{aligned}
$$

From (14), it follows that $1-\varphi>0$. Hence, the fixed point Problem (3) has a unique solution and consequently the iterative solution $u_{n+1}$ obtained from algorithm 6 converges to the exact solution $u$ of (1).

Theorem 3. Let the operator $T$ be strongly monotone with constant $\alpha>0$ and Lipschitz continuous with constant $\beta>0$. If there exists a constant $\rho>0$ such that

$$
\begin{aligned}
& 2 \alpha \rho-\beta^{2} \rho^{2} \\
& -(\mu+\lambda \rho \beta)\left(1+\sqrt{1-2 \alpha \rho+\beta^{2} \rho^{2}}\right)>0
\end{aligned}
$$

then, the approximate solution $u_{n+1}$ obtained from Algorithm 11 converges strongly to the exact solution $u \in K$ and satisfying the variational inequality (1).

Proof. Let $u \in K$ be a solution of (1) and $u_{n+1}$ be the approximate solution obtained from Algorithm 11. Then, from (3), (9) and (13), we have

$$
\begin{aligned}
\left\|u_{n+1}-u\right\| \leq & \mu\left\|u_{n}-y_{n}\right\|+\left\|y_{n}-u-\rho\left(T y_{n}-T u\right)\right\| \\
& +\lambda \rho\left\|T y_{n}-T u\right\|+\lambda \rho\left\|T u_{n}-T u\right\| \\
& \leq \mu\left\|y_{n}-u\right\|+\mu\left\|u_{n}-u\right\|+\theta\left\|y_{n}-u\right\| \\
& +\lambda \rho \beta\left\|y_{n}-u\right\|+\lambda \rho \beta\left\|u_{n}-u\right\| \\
& \leq\left[\mu \theta+\mu+\theta^{2}+\lambda \rho \beta(\theta+1)\right]\left\|u_{n}-u\right\| \\
& =\left[(\mu+\lambda \rho \beta)(\theta+1)+\theta^{2}\right]\left\|u_{n}-u\right\| \\
& =\varphi\left\|u_{n}-u\right\|,
\end{aligned}
$$

where

$$
\begin{aligned}
\varphi & =(\mu+\lambda \rho \beta)(\theta+1)+\theta^{2} \\
& =(\mu+\lambda \rho \beta)\left(1+\sqrt{1-2 \alpha \rho+\beta^{2} \rho^{2}}\right)+1-2 \alpha \rho+\beta^{2} \rho^{2}
\end{aligned}
$$

From (15), it follows that $1-\varphi>0$. Hence, the fixed point Problem (3) has a unique solution and consequently the iterative solution $u_{n+1}$ obtained from algorithm 11 converges to the exact solution $u$ of (1). $\square$

\section{Conclusion}

In this paper, we have used the equivalence between the variational inequality and the fixed point problem to suggest and analyze some new implicit iterative methods for solving the variational inequality. We also show that the new implicit methods includes the extra gradient method of Korpelevich [3], the modified extra gradient method of Noor [6], the proximal point methods of Noor et al. [4], and the unified implicit methods of Noor et al. [5] as special cases. We also have discussed the convergence analysis of the proposed new iterative methods under some suitable conditions. One may modify again this algorithmic schemes by different choices and rearrangement of the values of $\lambda$ and $\mu$.

\section{Acknowledgements}

The author would like to express her thanks to the anonymous referee for his valuable comments to improve the final version of this paper.

\section{REFERENCES}

[1] G. Stampacchia, "Formes Bilineaires Coercitives Sur Les Ensembles Convexes," Académie des Sciences de Paris, Vol. 258, 1964, pp. 4413-4416.

[2] J. L. Lions and G. Stampacchia, "Variational Inequalities," Communications on Pure and Applied Mathematics, Vol. 20, No. 3, 1967, pp. 493-512. doi:10.1002/cpa.3160200302

[3] G. M. Korpelevich, "An Extragradient Method for Finding Saddle Points and for Other Problems," Ekonomika i Matematicheskie Metody, Vol. 12, No. 4, 1976, pp. 747756. 
[4] M. A. Noor, K. I. Noor and E. Al-Said, "On New Proximal Methods for Solving the Variational Inequalities," Journal of Applied Mathematics, 2012, pp. 1-7.

[5] M. A. Noor, K. I. Noor, E. Al-Said and S. Zainab, "Study on Unified Implicit Methods for Solving Variational Inequalities," International Journal of Physics, Vol. 7, No. 2, 2012, pp. 222-225.

[6] M. A. Noor, "Some Developments in General Variational Inequalities," Applied Mathematics and Computation, Vol. 152, No. 1, 2004, pp. 199-277. doi:10.1016/S0096-3003(03)00558-7

[7] M. A. Noor, K. I. Noor and T. M. Rassias, "Some Aspects of Variational Inequalities," Journal of Computational and Applied Mathematics, Vol. 47, No. 3, 1993, pp. 285-312.

[8] D. Kinderlehrer and G. Stampacchia, "An Introduction to Variational Inequalities and Their Applications," Society for Industrial and Applied Mathematics (SIAM), Phila- delphia, 2000. doi:10.1137/1.9780898719451

[9] E. Al-Shemas, "Wiener-Hopf Equations Technique for Multi-Valued General Variational Inequalities," Journal of Advanced Mathematical Studies, Vol. 2, No. 2, 2009, pp. 01-08.

[10] E. Al-Shemas and S. Billups, "An Iterative Method for Generalized Set-Valued Nonlinear Mixed Quasi-Variational Inequalities," Journal of Applied Mathematics, Vol. 170, No. 2, 2004, pp. 423-432. doi:10.1016/j.cam.2004.01.028

[11] E. Al-Shemas, "Projection Iterative Methods for MultiValued General Variational Inequalities," Applied Mathematics \& Information Sciences, Vol. 3, No. 2, 2009, pp. 177-184.

[12] E. Al-Shemas, "Resolvent Operator Method for General Variational Inclusions," Journal of Mathematical Inequalities, Vol. 3, No. 3, 2009, pp. 455-462. doi:10.7153/jmi-03-45 